AUTHOR(S) Rhon Kelnigs, Michael E. Jones, and .J. J. Su

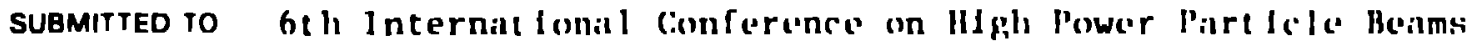
Kolse, Japin, Junc 9-12, 14 Ho

\title{
IMIST 'I.AIMF.K
}

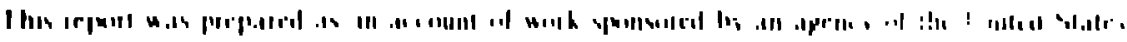

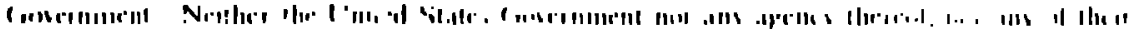

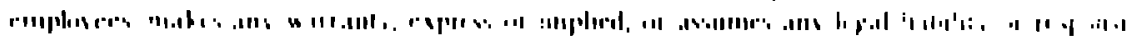

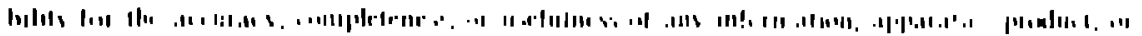

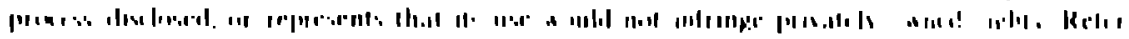

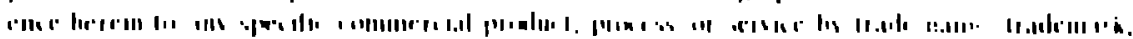

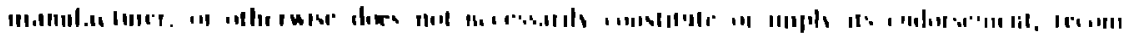

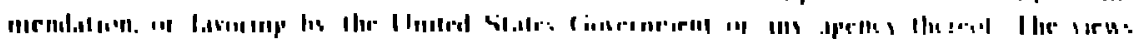

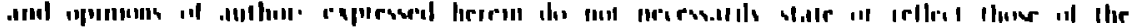

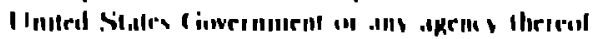

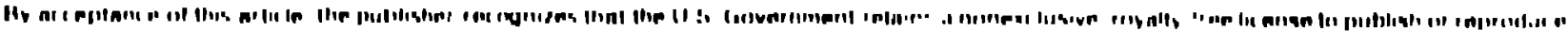

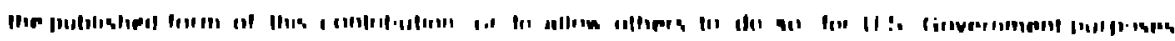

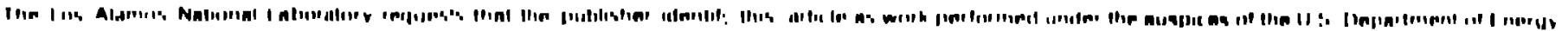

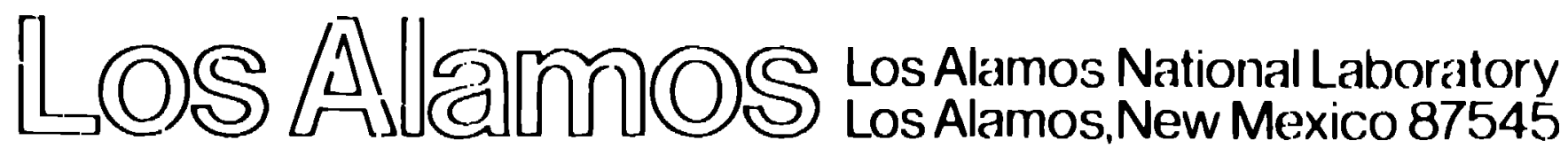

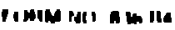




\author{
Rhon Keinigs and Michael E. Jones \\ Los Alamos National Laboratory \\ Los Alamos, New Mexico, 87545 \\ and \\ J.J. Su \\ University of Califomia Los Angeles \\ Los Angeles, California: 90024
}

\begin{abstract}
Introduction
We present an investigation of the two-dimensional dynamics of the plasma wakefield accelerator (PWFA) [1]. The PWFA is an advanced accelerator concept based on utilizing a large amplitude plasma wave to accelerate relativistic electrons to ultra-high energy. There are several reasons for using a plasma as an accelerating medium: (1) since the plasma is fully ionized there are no problems associated with breakdown, (2) a plasma is a single mode structure, so there are no parasistic losses due to the excitation of multiple modes, and (3) the accelerating fields produced by a plasma wave can be extremely large. Defining $n_{p}$ and $\delta n$ to be the background plasma density and perturbed density respectively. one finds the following scaling for the eleitric field,

$$
\mathrm{E}_{\mathrm{a}}-100 \mathrm{Gev} / \mathrm{m}\left(\frac{n_{p}}{10^{18}}\right) \frac{\delta n}{n_{p}} .
$$

llence, one finds that in a plasma of density, $n_{p}=10^{14}$, a wavebreaking field of a Gev/m is achievable.

The plasma tave represents the wake produced by a relativistic beam as it passes through a cold plasma. As the beam enters the plasma its space charge polarizes the background. When the beam tail passes the plasma is left with a large induced charge that oscillates at the plasma frecpuency, $(1)_{p} \quad\left(4 \pi c^{2} n_{p} / m\right)^{1 / 2}$. This disturbance follows along behind the beam, therefore its phase velexity equals the beam velocity (iiven $v_{p h}\left(0_{p} / k\right.$, onc finds the wavelength for this electrostatic perturbation is $\lambda_{p}$, $2 \pi v_{h} /(1)_{p}, \cdots 2 \pi c /(1)_{p}$,

The efficiency of any wakefield accelerator is determined by flic transformer ratto. Ihis is defined an the rattio of the maximum acceleratting field behind the
\end{abstract}


driving beam and the maximum decelerating field within the beam, $R=E_{a} / E_{d}$. In order to have an efficient accelerator $R$ must be large. Bane, et. al. [2] have demonstrated that large transformer ratios can be achieved by properly ramping the beam current. Maintaining a large a transformer ratio requires that this optimal current distribution remains unaltered during the entire acceleration phase. Results of our two-dimensional analysis and simulations show that this is not the case.

\section{Derivation uf the Wakefields}

The driving current distribution is assumed to be an arbitrary function of radius and the variable, $y=v_{b} t-z$, where $v_{b}$ is the beam velocity. The head of the beam corresponds to $y=0$, and the tail of the beam corresponds to $y=L$. The wave equation for the electric field can be expressed as

$$
\left(\nabla^{2}-\frac{1}{c^{2}} \frac{\partial^{2}}{\partial t^{2}}\right) E(r, t)=\frac{4 \pi \partial}{c^{2} \partial t} j+4 \pi \nabla p
$$

where $\mathbf{j}$ and $\rho$ are the total current and charge densities. Separating the source term into (wo pieces, an exiernal beam contribution represented by the subscript, b, and an induced plasma response denoted with a subscript, p, Eq. (1) can be expressed in the form of a K'inc-Gordon equation. Using Ohm's law to eliminate the plasma current from the RIIS of liq. (I) in favor of $\mathrm{F}$ we obtain

$$
\left(\nabla^{2}-\frac{c p_{p}^{2}}{c^{2}} \cdots \frac{1}{c^{2}} \frac{\partial^{2}}{\partial t^{2}}\right) \mathbf{k} \quad \frac{4 \pi}{c^{2}} \frac{\partial}{\partial t} j_{h}+4 \pi \nabla_{p_{l 11}}
$$

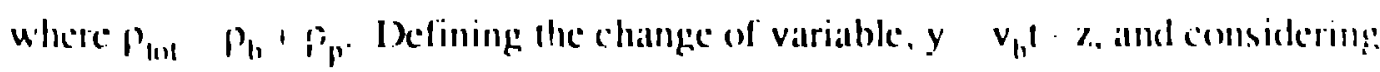
the source w be itrimuthally symmetric, this eefuition becomes 


$$
\begin{aligned}
\left(\Delta-k_{0}^{2}+\frac{1 \partial^{2}}{\gamma^{2} \partial y^{2}}\right) E= & -z 4 \pi \frac{\partial}{\partial y}\left(\frac{\rho_{b}}{\gamma^{2}}+\rho_{p}\right) \\
& +r 4 \pi \frac{\partial}{\partial r}\left(\rho_{b}+\rho_{p}\right) .
\end{aligned}
$$

A similar equation for the magnetic field is obtained from Eq. (3) and Faraday's law;

$$
\left(\Delta-k_{0}^{2}+\frac{1 \partial^{2}}{\gamma^{2} \partial y^{2}}\right) B=\theta 4 \pi \beta \frac{\partial}{\partial r} \rho_{b}
$$

Here we have substituted $j_{b}=\rho c \rho_{b}, \gamma=\left(1-\beta^{2}\right)^{-1 / 2}$, and have defined $\Lambda \therefore \nabla^{2}$. $\partial^{2} / \partial z^{2}, k_{0}=\omega_{p} / c$. These equations are solved by Fourier-transforming in the variable, $y$, and Hankel transforming in the radial variable. The induced chargo density is eliminated in favor of the beam charge density using the continuity equation coupled with Poisson's equation. The following solutions are exact to order $\gamma^{2}$. In front of the beam $(y<0)$ all field components. $E_{z}, E_{r}$, and $B_{f}$. vanish identically. The magnetic field also vanishes behind the beam. Within the beam

$$
\begin{aligned}
& E_{2}(r, y) \quad-4 \pi k_{r}^{2} \int_{0}^{\infty} d r^{\prime} r^{\prime} \sigma\left(r^{\prime}\right) I_{0}\left(k_{p} r \cdot\right) k_{0}\left(k_{p} r\right) \\
& \cdot \int_{0}^{y} d y^{\prime} f\left(y^{\prime}\right) \cos k_{p}\left(y \cdot y^{\prime}\right) \\
& I_{r}(r, y) \quad 4 \pi \int_{0}^{\infty} d r^{\prime} r^{\prime} \frac{d \sigma}{d r^{\prime}} I_{1}\left(k_{p^{\prime}} r\right) k_{!}\left(k_{r^{\prime}} r^{\prime}\right)
\end{aligned}
$$




$$
\begin{gathered}
\left.\cdot\left(k_{p} \int_{0}^{y} d y^{\prime} f\left(y^{\prime}\right) \sin k_{p} y-y^{\prime}\right)-f(y)\right) \\
B_{\theta}(r, y)=-4 \pi \beta \int_{0}^{\infty} d r^{\prime} r^{\prime} \frac{\partial \sigma}{\partial r^{\prime}} I_{1}\left(k_{p} r_{a}\right) k_{1}\left(k_{p} r\right) \cdot f^{\prime}(y) .
\end{gathered}
$$

In Equations (5) - (7) $I_{v}$ and $K_{v}$ are the usual modified Cessel functions of order, $v$. and $r_{s}$ and $r_{\text {, }}$ respectively denote the minimum and maximum of $r$ and $r^{\prime} ; k_{r}=$ $\left(\omega_{p} / v_{b}\right.$. Behind the beam $(L<y)$ the upper limit on the $y$-integrations gits replaced by $L$, and the second term in brackets in the equation for $E_{r} \alpha f(y)$ is absent. Note that in this region $E_{z}$ and $E_{r}$ are oscillatory and nincty degrees out of phasc, meaning that there are forty-five degrees of phase in which a trailing bunch can be both accelerated and forused.

Current shaping has a signiiicant effect on the wakelields produced within be:am. To study this we let $p_{h}(r, y) \quad \sigma(r) f(y)$. We alssume that the radial current profile is at dexorstep distribution, $\sigma(r) \quad \sigma_{0}(-)(a-r)$. and focus our altention on the axial profile, $f(y)$. We analyze the current profile first studied by Bane |2|. This profile results in an axial decele rating field within the beam that is uniform, and ceads to the latgess value for the transformer ratio. In addition lo having a special. distribution the current is cut off abruptly at the tail of the beam. If the current is not cut off in a time that is short compared to a plasma period. (1) '. then the plasma clectroms will have time to relax batck to their unperturled pesitions and the watefich amplitude will be reduced! [3]. This is a severe fechnical limitattion on achieving hipgl, accelerating gradiants in the plasma watkelicld accelerator. For the calse of the "phimal current profile. 


$$
\begin{aligned}
f(y) & =\frac{1}{1-\frac{\pi}{2}+k_{p} L} & 0 \leq y \leq \lambda_{p} / 4 \\
& =\frac{1-\frac{\pi}{2}+k_{p} y}{1-\frac{\pi}{2}+k_{p} l .} & \lambda_{p} / 4 \leq y \leq L .
\end{aligned}
$$

The fields behind the doorstep $\left(0 \leq y \leq \lambda_{p} / 4\right)$ are

$\lambda_{p} / 4 \leq y \leq L$

$$
E_{,}(r, y)=-\frac{4 \pi \sigma_{0}}{k_{r}} R_{1}(r) \frac{1}{1-\frac{\pi}{2}+h_{p} L}
$$

$$
E_{r}(r, y) \quad 0
$$

$$
B_{0}(r, y)-\frac{4 \pi \beta \sigma_{0}}{k_{p}} R_{2}(r) \frac{1}{1-\frac{\pi}{2}+k_{p} l}
$$

1. $\because y$

$$
\begin{aligned}
& l_{,}(r, y) \quad \frac{4 \pi \sigma_{0}}{k_{1}} R_{1}(r)\left(\sin k_{p} y \cdot \frac{\cos k_{p}(y-l)}{1 \frac{\pi}{2}+k_{p} I}\right) \\
& I_{1}(r, y) \quad \frac{4 \pi \sigma_{(1)}}{k_{r}} k,(1)\left(\cos k_{p} y+\frac{\sin k_{p}(y-1)}{1 \frac{\pi}{2}, k_{p} I}\right) .
\end{aligned}
$$


The racial dependencies are given by

$$
\begin{aligned}
& R_{1}(r)=1-k_{p} a K_{1}\left(k_{p} a\right) I_{0}\left(k_{p} r\right) \\
& R_{2}(r)=k_{p} a K_{1}\left(k_{p} a\right) I_{1}\left(k_{p} r\right) .
\end{aligned}
$$

From the equations for the axial electric field one finds that $R=1-\pi / 2+k_{p} L$ for this distribution For long pulse lengths $R>1$. However, the beam pinching for this profile is not uniform along the beam axis, and this will eventially lead to reduction in $R$. Behind the doorstep the radial wakefield, $W-E_{r}-\beta B_{\theta}$, is solely duc to the azimuthal B-ficld;

$$
W^{\prime}(r, y)=-\frac{4 \pi \beta \sigma_{0}}{k_{p}} R_{2}(r) \frac{1-\frac{\pi}{2}+k_{p} y}{1-\frac{\pi}{2}+k_{p} L}
$$

\section{Self-consistent Evolution of the Driving Bunch}

The foregoing analysis has yielded the longitudinal and transverse wakeficlds assuming that the charge and current profiles of the driving bunch remain fixed. If tailoring of the driving bunch is to make sense, the beam must lose most of its energy before the 'ransverse wakefield alters the profile of the tailored bunch. $\Lambda_{n}$ estimate of the condition necressary for maintaining the proíte of the driving bunch can be made by comparing the ratio of the longitudinal wakefield to the transverse wikefield within the beam. Aside from factors which depend on the detailed axial profile of the bunch this ratio is given by 


$$
\frac{W}{E_{z}} \sim \frac{R_{2}(r)}{R_{1}(r)}=\frac{k_{p} a K_{1}\left(k_{p} a\right) I_{1}\left(k_{p} a\right)}{1-k_{p} a K_{1}\left(k_{p} a\right) I_{0}\left(w_{p} i\right)}
$$

For beam pulse shaping to have an effect this ratio must be small. The doorstep profile makes this ratio larger by the transformer ratio. For beams in which the radius is small $\left(k_{\mathrm{p}} \mathrm{a} \ll 1\right) \mathrm{W} / \mathrm{E}_{\mathrm{z}} \gg>1$ except very near $\mathrm{r}=0$. Thererfore, the transve $e_{s e}$ wakefield will always dominate the longitudina! wakefield and pulse tailoring will be ineffective. For large radii beams $W / E_{z}$ can be made large everywhere except within a couple of skin depths $\left(c / \omega_{p}\right)$ of the beam edge. The difference between the small and large radii beams is attributahle to a difference in the return curreuis induced in the plasma.

We now give an estimate of the current required to result in an accelerating field that approaches the waveureaking limit. From Eq. (113) the electric field behind the driving bunch can be scaled in terms of dimensionless variables as follows.

$$
\frac{\mathrm{el}}{\mathrm{mc} \omega_{\mathrm{p}}}=\frac{4\left(\mathrm{el} / \mathrm{mc}^{3}\right)}{\left(\omega_{\mathrm{p}} \mathrm{a} / \mathrm{c}\right)^{7}}
$$

where $\mathrm{I}$ is the peak current in the beam. Note that the dimensionless quantity on the left-hand side of the equation is the peak electric ficld normalized to the wave breaking field. It is desirable to have this quantity near one. However, the requirement that the beam be large in order to maintain palse tailoring means that the peak current must be large. This makes it very difficult to achieve the maximiun accelerating field possible in a plasma. Futhermore, large currents tend to make the driving beam unstable to filamentation and hose instabilities.

To test these conclusions and to explore more fully the self-consisitent evolution of the driving beam in a plasma wakefield accelerator, the system has been simulated with the particle in cell model, ISIS [4]. Simulations have been periormed with immobile ions and particle plasina electrons and particle beam electrons.

The beaun dynamics for a small beam $\left((1)_{p} \mathrm{a} / \mathrm{c} \quad 2, \mathrm{l}_{\mathrm{pk} \text { :ak }} \quad 3.4 \mathrm{k} \Lambda\right)$ are shown in ligh. (1). The beam particles are injected from the keft-hand boundary to give an 
optimal transformer profile, with a transformer ratio of five. The peak injected beam density is $n_{b} / n_{p}=0.2$, and the team energy, $m^{2} c^{2}=20$. The figure consists of five snapshots of the beam particle positions superimposed. By the second snapshot the pinching has become severe. The fact that the pinching is nonuniform means that the doorstep pulse tailoring is destroyed by this time. The phase space for the bunch is shown in Fig. (2). As can be seen from this figure the energy loss is very nonuniform, meaning a loss of accelerator efficiency. Fig. (3) shows the same simulation as Fig. (1) for a beam radius 10 times larger $\left(I_{\text {peak }}=340 \mathrm{kA}\right)$. As predicited, initially the pinching occurs only near the beam edge. Comparing Fig. (3) with Fig. (1) shows that the larger beam maintains its integrity longer than the smaller beam. However, the large beam is seen to undergo a strong filamentation instability, resulting in the nonuniform deceleration displayed in the phase space plot shown in Fig. (4).

These st'dies indicate that the self-consistent transverse motion of the driving bunch makes pulse tailoring impossible to maintain. If the beam density is reduced so that the accelerator is operated well below wavebreaking ( $\left.\mathrm{eE} / \mathrm{mc} \omega_{\mathrm{p}} \ll 1\right)$, less current is be needed to avoid beam pinching. At higher voltages, the beam pinching will be slower because relativistic effects make the beam more massive and hence stiffer. However, the beam must also travel farther in order to lose its energy. 13oth of these effects scale as the Lorentz factor, $\gamma$. Thus the pinching cannot be ameliorated by using higher energy beams.

An altemative method of preventing beam pinching is to apply an extemal axial magnetic field. A conservative estimate of the magnetic ficld required can be obtained by assuming that $\Omega_{\mathrm{c}}>(1)_{\mathrm{p}}$. To get an accelerating gradient of $1 \mathrm{Gev} / \mathrm{m}$, the plasma frequency must be at least $\omega_{p}=5.64 \times 10^{11} \mathrm{sec}^{-1}$, which means a magnetic field, $\mathrm{B}_{z} \approx 30 \mathrm{kG}$, must be used in order to avoid bean pinching. The complic ition of applying a magnetic fiela of 10 's of kilogauss along the acceicrator places at severe technological restriction on the acceleratin i? gradient that can be obtained in a plasmal wakefield accelerator. Fïg. (5) shows the same simulation as Fig. (1) but with $\Omega_{c} /(1), \quad 5.0$. 


\section{Refereinces}

1. P. Chen, J.M. Dawson, R.W. Huff and T. Katsouleas, Phys. Rev. Lett. 54, 693 (1985)

2. K.L.F. Bane, P. Chen and P.B. Wilson, IEEE Transactions on Nuclear Sci., Vol. NS-32, No.5, 3524 (1985)

3. T. Katsouleas, Phys. Rev. A. 33, No.3, 2056 (1986)

4. G. Gisler and M.E. Jones, in Proc, of the 11th Intl. Cont, on Numerical Sirulations of Plasmas, Montreal, Quebec (1985) 

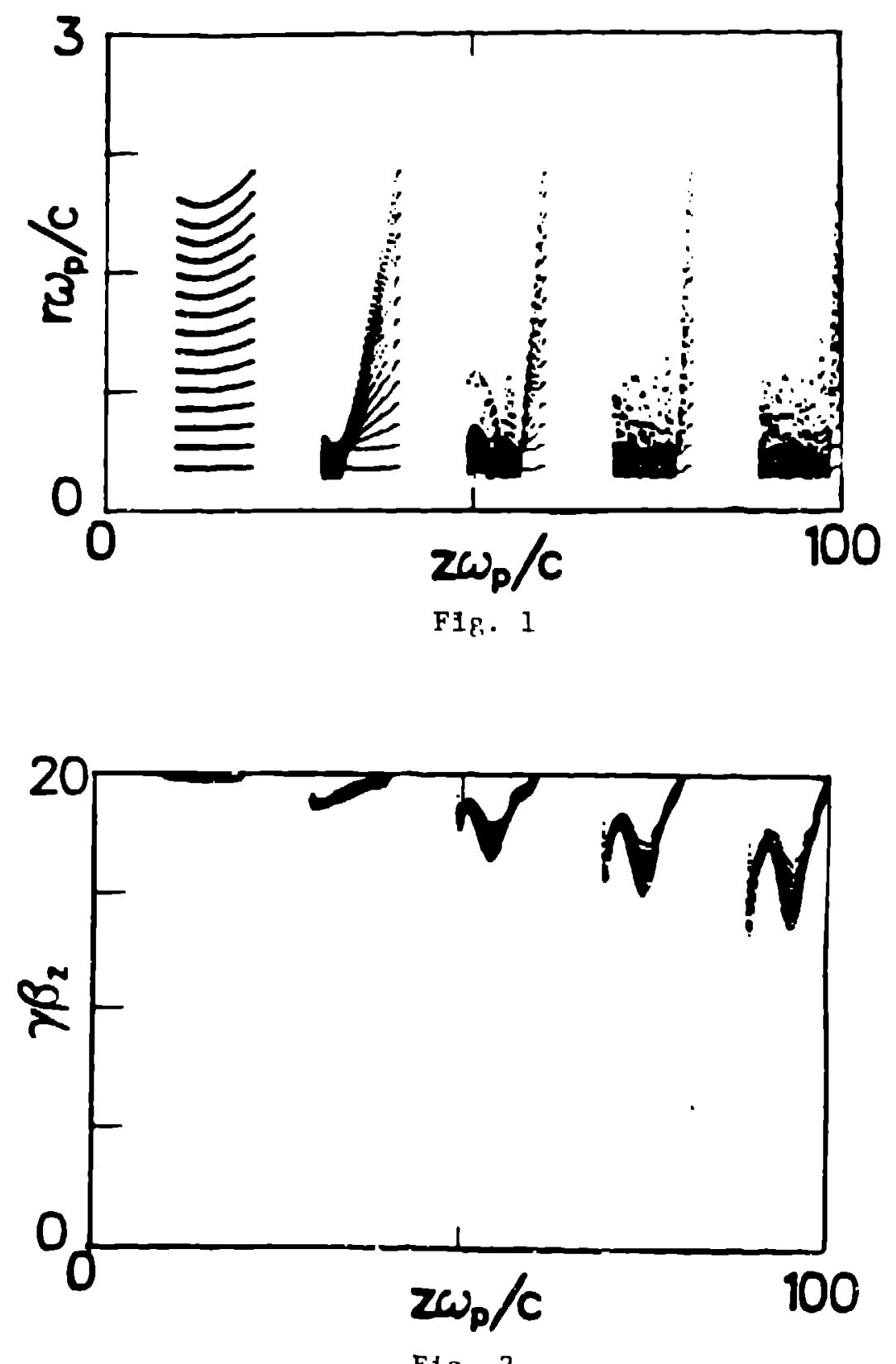

F」g. 2

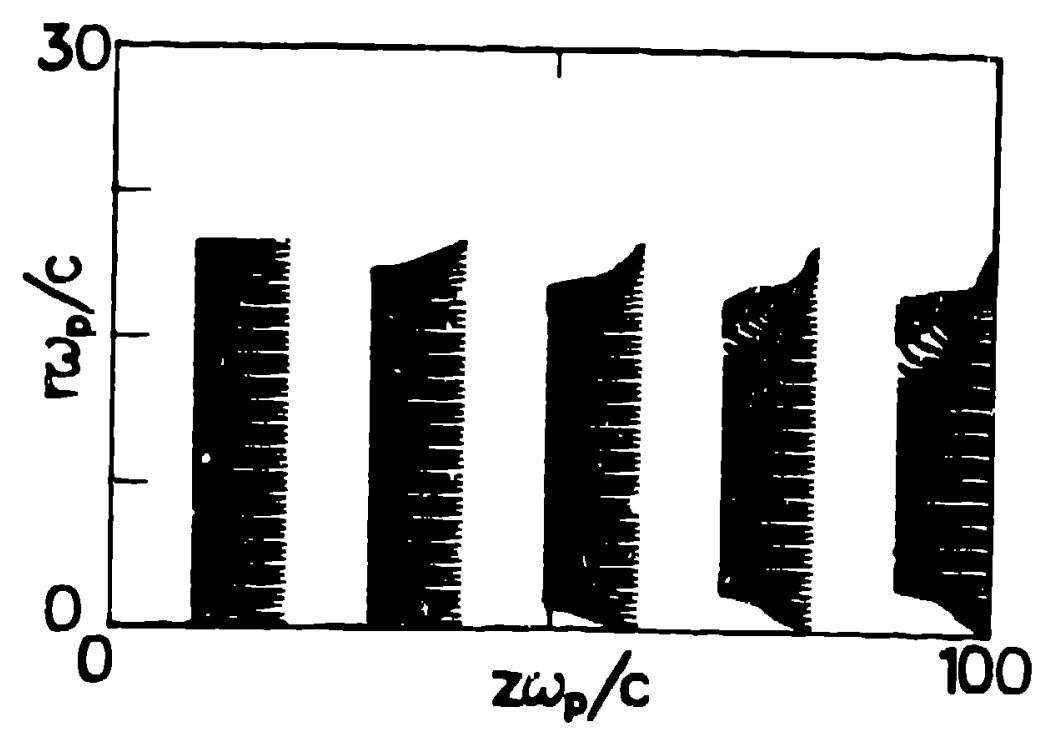

F1p. 3 


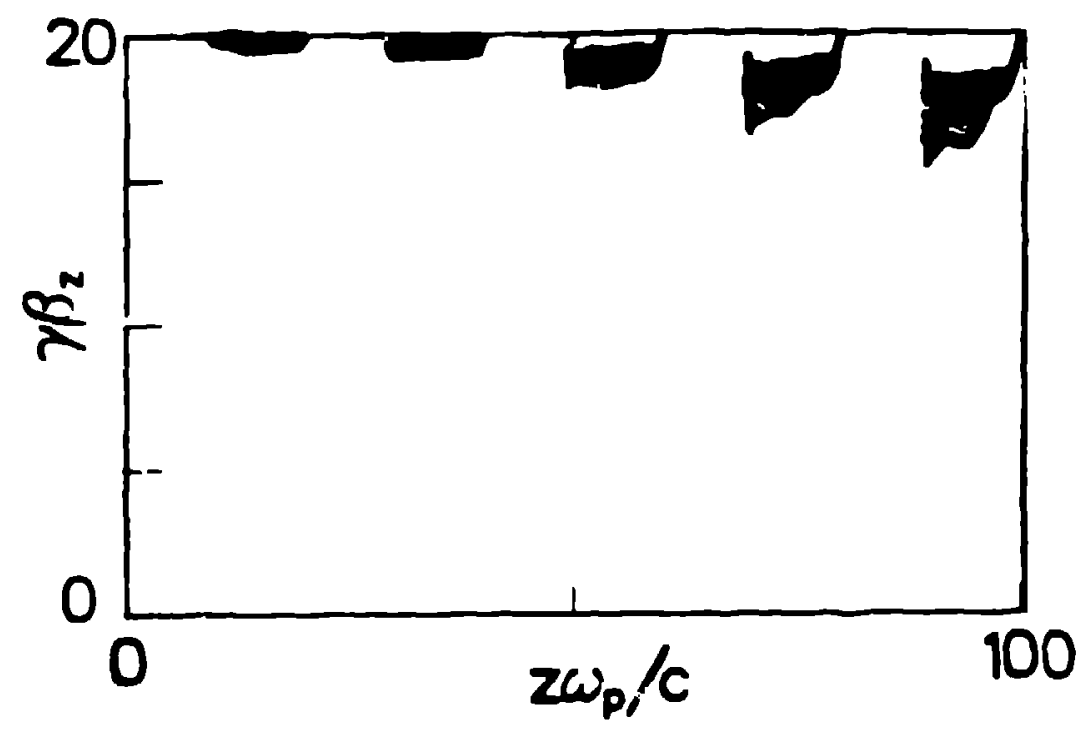

Fig. 4

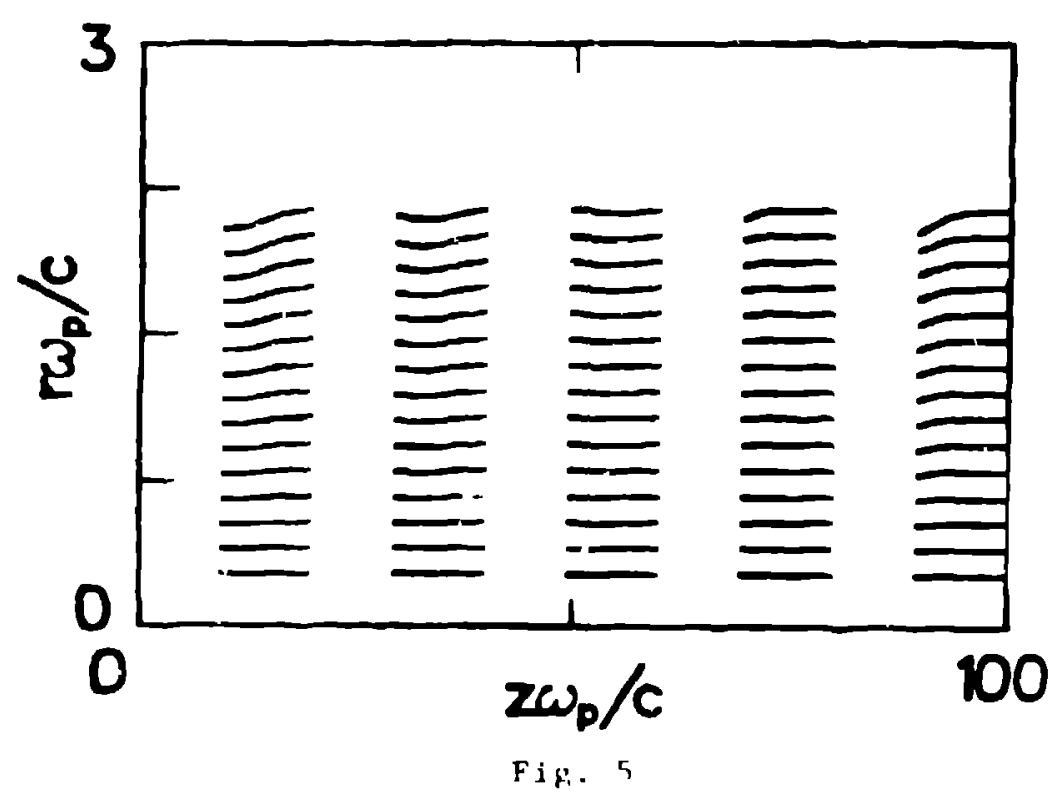

